Journal of Engineering and Applied Sciences 14 (15): 5287-5296, 2019

ISSN: 1816-949X

(C) Medwell Journals, 2019

\title{
An Energy Efficient Cluster based Routing Protocol for MANETs using DCCHSM Algorithm
}

\author{
J. Vijayalakshmi and K. Prabu \\ Department of Computer Science, Sudharsan College of Arts and Science, \\ Pudukkottai, 622104 Tamil Nadu, India \\ kv.anandeesh@gmail.com, kprabu.phd@gmail.com
}

\begin{abstract}
MANETs or Mobile Ad hoc Networks are computer networks that have mobile devices, self-arranged functioning without infrastructure and a wireless medium for information distribution. MANET's have extraordinary characteristics include dynamic network topology and moving nodes. MANET is very helpful in regions for networks that require ad hoc connectivity and have devices with mobility. This sudden spurt in MANETs has attracted research in suggesting optimal routing schemes for MANETs. Further, MANETs are also vulnerable to attacks of several kinds. Further, the need for better speed and efficiency in these mobile devices keeps rising every year and the resulting demand for further protocol optimization is seen. This study proposes an DCCHSM, a hybrid technique using k-means and k-medoids for secure routing efficiency in MANETs. This study also displays the results of DCCHSM efficiency in its implementation.
\end{abstract}

Key words: MANET, routing protocols, MANET applications, DCCHSM, clustering nodes, routing, security

\section{INTRODUCTION}

MANET is a wireless network in which every device liberated to move not dependently in some direction (Schiller, 2003). These network devices change positions resulting in a vastly dynamic where each one of them participate as a router of the network. The life cycle of MANET can be characterized into earliest, next and third generation (Basagni et al., 1998; Eichler and Roman, 2006). MANETs roots able to be outlined back in the 1970 's to Packet Radio Networks (PRNETs) from Defense Advanced Research Project Agency (DARPA) research which used a packet-switched radio communication to provide consistent communique among computers and urban population. It evolved into Survivable Adaptive Radio Network (SURAN) in the early 1980's (Chen and Gerla, 1998). SURAN improved radio performances by making them lesser, cheaper and power thrifty. SURAN also provides flexibility to electronic attacks. MANET was born in Internet Engineering Task Force (IETF) which standardized routing protocols for MANET and gave rise to the development of various mobile devices. The development of standard IEEE 802.11 for WLAN's also benefited MANET. The applications of MANET are communication, conference meeting, create effective classrooms and military and in the wireless sensor network. While changing the network condition at the same time through the strategy of routing used as the limited resources such as: network size, partitioning andtraffic size. It provides a different quality of services to a different users and applications. A routing protocol is required while a packetis needed be broadcast to a destination. These protocols tofinding a path for packet rescue and transferred the packet to the destination. Routing techniques are needed to send data from nodes to sink nodes by establishing communication. Routing in a MANET may vary based on the application and routing problems lead to decreased network lifetime with increased energy consumptions. Many protocols have been urbanized for minimizing energy consumptions and can be categorized based on the node's participation, functioning modes, clustering and network structures. Packets are relayed directly from one intermediate node to the next. The plan and execution of routing methods that are able to effectively and proficiently support information exchange and processing in MANET is a complex task (Al-Karaki and Kamal, 2004). This study proposes a routing protocol called Energy Efficient Cluster based Routing Protocol (DCCHSM) for energy efficiency and speed in routing of information in a MANET.

Issues and design challenges in MANET routing: MANET issues start with tracing the destination when a

Corresponding Author: J. Vijayalakshmi, Department of Computer Science, Sudharsan College of Arts and Science, Pudukkottai, 622104 Tamil Nadu, India, kv.anandeesh@gmail.com 
node moves in a MANET, its address gets changed and packets contain a header with its previous address. The sudden change in IP address is not accepted by higher level protocols resulting in a loss of a connection. However, mobile IP, DHCP, cellular networks have been developed to support mobility as they use wireless communication without any infrastructure. These networks have succeeded when telecommunication infrastructure is lean, incurring a minimum cost information share in varied geographical locations. Certain issues that have been causing concern in MANETs are security and mutual trust, leaving them open to attacks. The mobile and dynamic network topology of a MANET also makes it complicated to trace a malicious node.

MANET routing: The basic aim of a routing protocol is to route information from a source to a destination by identifying the correct route. A major hurdle in routing is the network characteristics which may have variations. MANET routing algorithms can be categorized as proactive, on-demand and hybrid routing algorithms. Proactive or table-driven algorithm's nodes have a routing table with information on nodes routing information and are similar to wired routing algorithms. The issue in this routing is delays caused in routing information updates, due to changes in MANET topology. Examples of proactive routing are DSDV (Destination-Sequenced Distance-Vector routing) (Perkins and Bhagwat, 1994) and an adapted Bellman-Ford algorithm (Bellman, 1957). These methods decrease the frequency of updates. Another proactive routing algorithm, CGSR (Cluster_head Gateway Switch Routing) (Chiang et al., 1997) used clustering for reducing update over heads. The study by Jacquet et al. (2001) OLSR (Optimized Link State Routing), exchanged link state information instead of looking at routes. On-demand routing initiates a path detection process from source for discovering a destination. The information, thus, transmitted is judged by a reply from the destination to the source. This technique creates only the required number of routes and helps reducing protocol over heads. Examples of this type of routing are AODV Ad hoc on-demand distance vector (Perkins and Royer, 1999) and DSR (Dynamic Source Routing) (Johnson and Maltz, 1996). Contrarily, ZRP (Zone Routing Protocol) (Pearlman and Haas, 1999) uses a two part approach where a node maintains routes of local zone using the proactive approach and distant destinations are discovered using the on-demand approach. Therefore, a hybrid protocol's characteristics would also

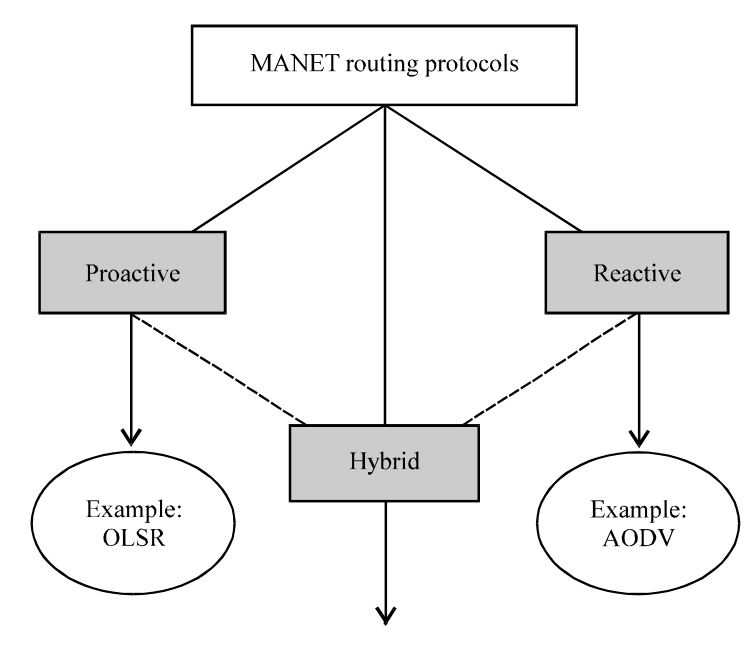

Fig. 1: MANET routing algorithms

be a combination of the properties of proactive and ondemand protocols. Figure 1 depicts MANET routing algorithms.

Existing studies have reflected the importance of clusters and clustering algorithms in choosing the shortest network path in a MANET network. k-means algorithm has been used as a cluster head selection method and can improve network performance of routing protocols (Ndlovu, 2015). This study used the concept of energy value of a sensor and evaluated routing paths for prolonging the network lifetime of the sensor. It introduced k-means algorithm for creating clusters and selecting the clustering heads in the network. The created cluster nodes were optimized by having the minimal distance from the cluster head.

DCCHSM: The goal of any routing protocol is finding an optimized and efficient path for information transfer between nodes. Most routing protocols are based on topology using existing network link information for packet forwarding. Current routing protocols utilizeposition information to locate destination and neighbor nodes for reliability. As straight transmission series is limited by radio proliferation effects mainly attenuation, a MANET does not form links with each other nodes in the network. Instead, all a small set of nodes are reachable, the neighbor set. Traffic from a source, destined for a node not in its neighbor set, must be ahead in a multi-hop manner as depicted in Fig. 2.

The proposed routing protocol DCCHSM is hierarchical in nature. One main feature of a MANET is that it is very different from other types of networks in its structure of the traffic pattern and may be query driven, event-driven, continuous monitoring and hybrid 


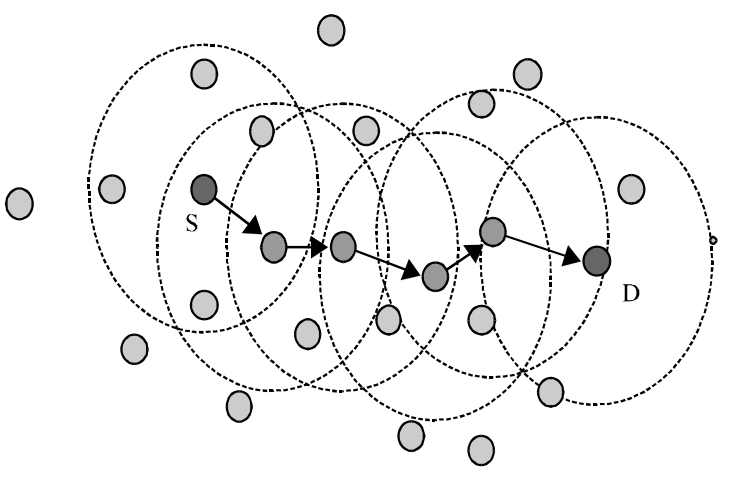

Fig. 2: MANET multi-hop traffic delivery

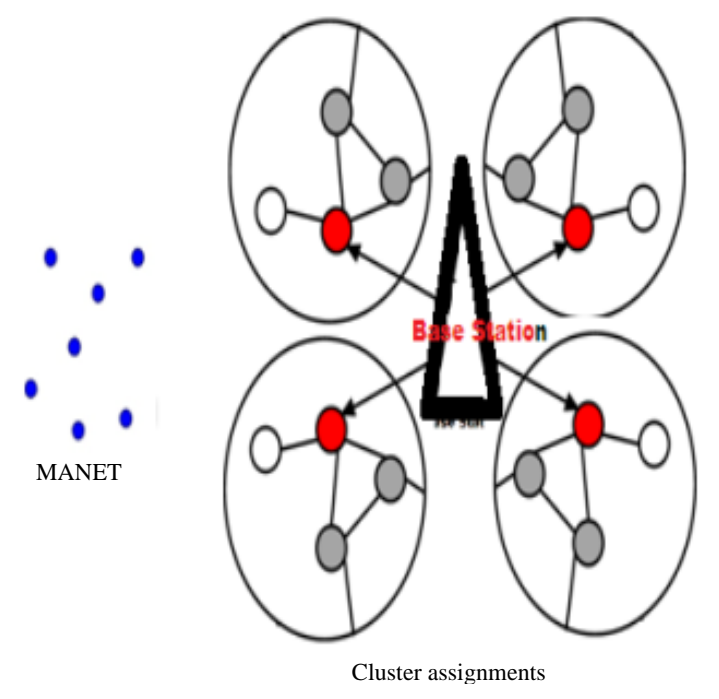

Fig. 3: Clustered MANET data flow

combination of previously stated traffic patterns (Vijayalakshmi and Prabu, 2018a, b). Traffic patterns significantly affect the choice of a routing protocol. For example, proactive approaches based on cyclic gathering of routing message for destination nodesis appropriate for unbroken traffic but need excessive energy. Figure 3 depicts the information flow in a clustered MANET.

\section{MATERIALS AND METHODS}

Workflow of proposed DCCHSM method: Figure 4 workflow of proposed energy efficient cluster based routing protocol. In DCCHSM first analysis the node in the topology then finding average distance of each node using shortest path (Dijkstra algorithm) with dynamic reconstruction graph. In the node connectivity is based on the minimum average distance of node to connect and construct the cluster group. Finding threshold of each neighborhood nodes in the network:

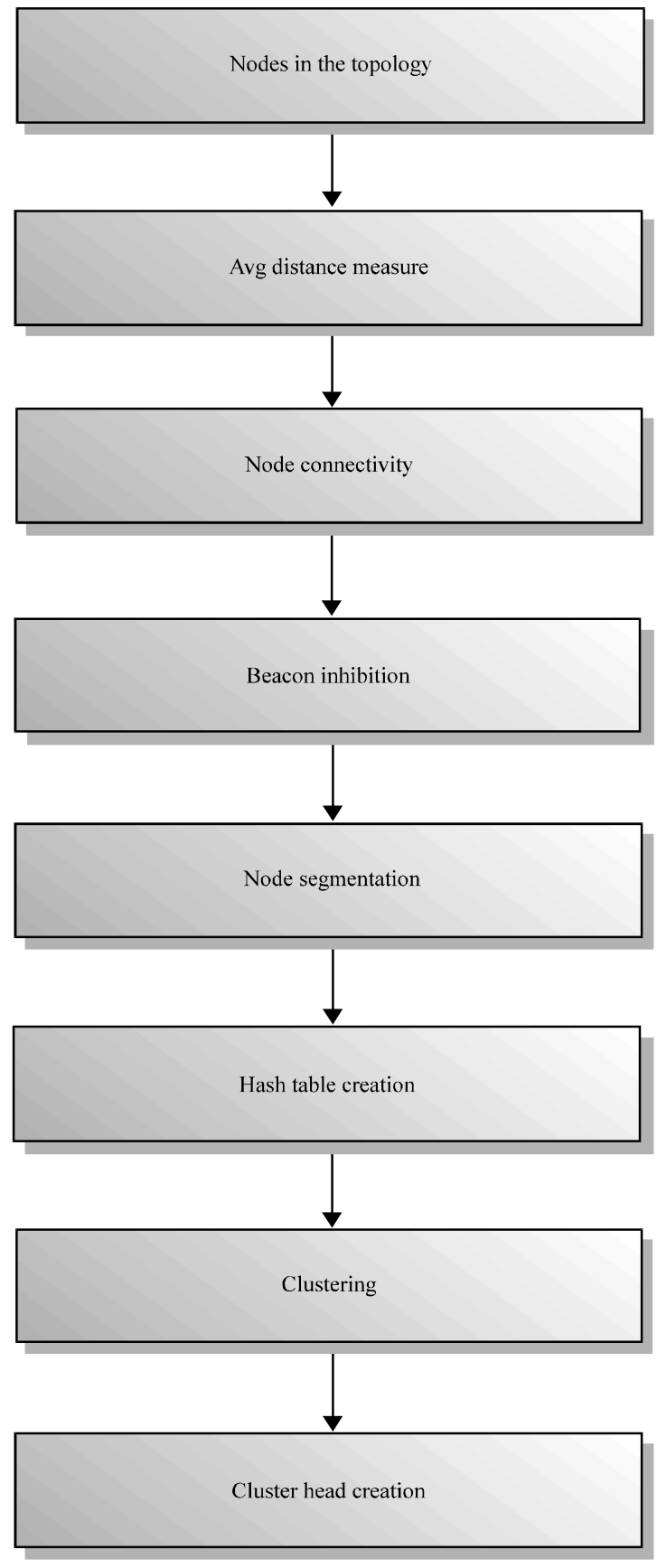

Fig. 4: Proposed workflow architecture

$$
\mathrm{T}=\frac{\text { Range of beacon }(\mathrm{R})}{\text { Time taken to send a beacon }(\mathrm{t})}
$$

In segmentation of nodes based on the minimum sensing threshold range in the network then neighbor table would created and updated with send HELLO packets to neighbor nodes related on the received packets to construct priority table for each node. 


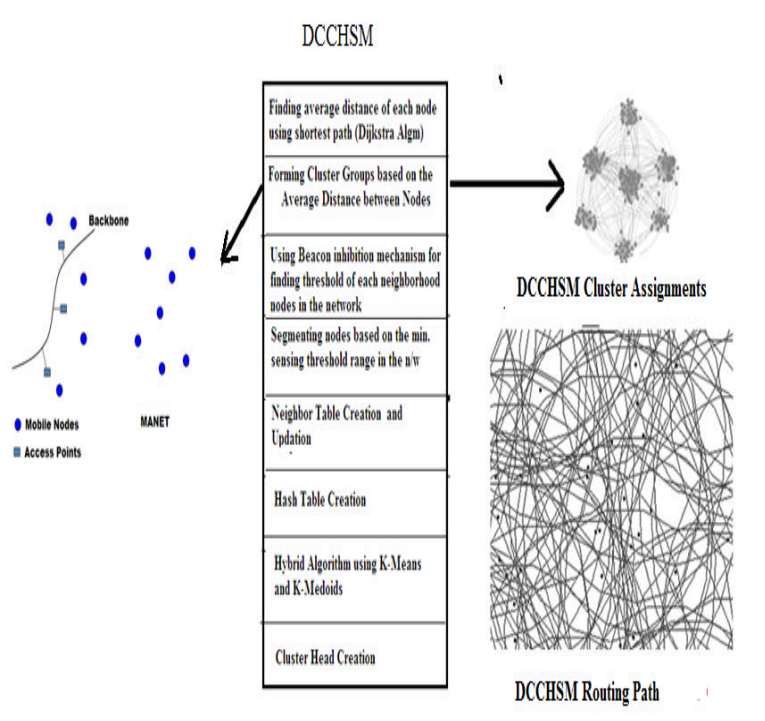

Fig. 5: DCCHSM architecture

In hash table creation neighbor table, if the nodes beacon value is less than average threshold value of node range, move nodes and construct hash table of each nodes. Finally, the clustering is applied based on the hybrid algorithm using k-means and k-medoids algorithms. In cluster group the average beacon value range node is form as a cluster head.

DCCHSM architecture: The proposed routing protocol DCCHSM is aimed at lesser energy consumption and faster information transfer between nodes. It uses hybrid clustering involving $\mathrm{k}$-means and $\mathrm{k}$-medoids for its information routing. It is an efficient protocol which defines its routing paths by detecting the numeral nodes in the network and using a distance measure to connect nodes and thus arrive at shortest routing paths between clusters. Figure 5 depicts the DCCHSM architecture.

DCCHSM in the initial step analyzes the amount of nodes in the network. It then finds the average distance between nodes using Dijkstra's algorithm and constructs a dynamic reconstruction graph based on the shortest path. This shortest path is then used for identifying nodes nearer to each other and for grouping them. Hierarchical protocols like DCCHSM split the network into zones or clusters (Abbasi and Younis, 2007). The nodes inside a cluster only required to deliver their data to the Cluster Head ( $\mathrm{CH})$. In turn, the $\mathrm{CH}$ can be component of a next level according to some hierarchical arrangement of the nodes rooted at the final sink nodes. The beacon inhibition mechanism is the used by DCCHSM to assess the time taken to transmit a beacon using Eq. 1 and also estimate the threshold values of the nodes:

$$
\mathrm{T}=\frac{\text { Range of beacon }(\mathrm{R})}{\text { Time taken to send a beacon }(\mathrm{t})}
$$

The estimated threshold value of a neighborhood (previously grouped nodes) is then segmented based on the threshold values. A neighbor table was created and priorities are updated by transmitting a HELLO packet to neighbor nodes. Hash table creation in DCCHSM is based on beacon values. In the neighbor table, if the nodes beacon value is less than average threshold value of node range then the nodes are moved for construction of the hash table of each node. Clustering is then applied to the node attributes using a hybrid technique involving k-means and k-medoids algorithms. Cluster assignments are then created using a cluster group's the average beacon value range of a node. The cluster assignment heads thus form the main points of action for DCCHSM protocol in MANET communications.

The following DCCHSM (Dynamic Clustering based Cluster Head Selection in MANET) algorithm is used to select the Cluster Head $(\mathrm{CH})$ in selected nodes for the proposed topology using cluster energy and cluster weight (Algorithm 1-4).

\section{Algorithm 1; DCCHSM algorithm:}

Input: Given the data points (node locations), choose the number of clusters $1<\mathrm{c}<\mathrm{N}$

Output: Selection of Cluster Head $(\mathrm{CH})$ from data points

Step 1: Initialize with random cluster centers chosen from the data set

Step 2: $\quad$ Repeat for $1=1 ; 2 ;::$ :

Step 3: Compute the distances:

$$
\mathrm{D}_{\mathrm{ik}}^{2}=\left(\mathrm{x}_{\mathrm{k}}-\mathrm{v}_{\mathrm{i}}\right) \mathrm{T}\left(\mathrm{x}_{\mathrm{k}}-\mathrm{v}_{\mathrm{i}}\right), 1 \leq \mathrm{i} \leq \mathrm{c}, 1 \leq \mathrm{k} \leq \mathrm{N}
$$

Step 4: Select the points for a cluster with the minimal distances they belong to that cluster

Step 5: Calculate cluster centers:

$$
\mathrm{v}_{\mathrm{i}}^{(1)}=\frac{\sum_{\mathrm{j}=1}^{\mathrm{N}} \mathrm{x}_{\mathrm{i}}}{\mathrm{N}_{\mathrm{i}}}
$$

Step 6: Until:

$$
\left.\prod_{\mathrm{k}=1}^{\mathrm{n}} \max \right|_{\mathrm{v}}(1)-_{\mathrm{v}}(1-1) \mid \neq 0
$$

Step 7: Calculate the partition matrix

Step 8: $\quad$ Set the point energies:

$$
\left.f(i)=\sum_{i=1}^{N} \text { point (1:dim_num, i)-cluster_center (1:dim_num, j }\right)^{2}
$$


Step 9: Set the cluster energies:

$$
\text { Cluster_energy }(j)=\text { Cluster_energy }(j)+f(i)
$$

Step 10: Adjust the point energies by a weight factor:

$$
f(i)=f(i) \frac{\text { cluster population }(j)}{\text { cluster population }(j)-1}
$$

Step 11: Assign weighted node $\mathrm{f}$ (i) as Cluster Head (CH)

Where, $\mathrm{x}=$ data point (node location); $\mathrm{v}=$ Neighbor distance node; $\mathrm{N}=$ Total number of nodes

\section{Algorithm 2; Pseudo code \\ Calculate the mean and sum of squares for each cluster:}

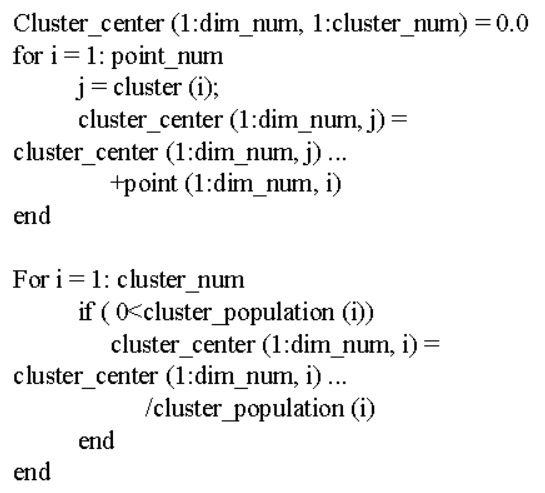

\section{Algorithm 3; Calculate point energies:}

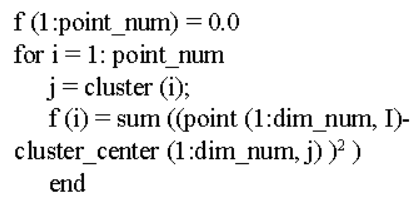

\section{Algorithm 4; Calculate Set the cluster energies:}

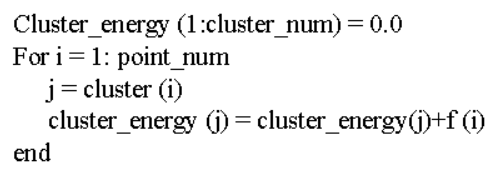

Table 1: Simulation parameters

\begin{tabular}{ll}
\hline Parameters & Values \\
\hline Simulation & NS-2 \\
MAC protocol & IEEE 802.11 \\
Protocol name & DCCHSM, DCH, \\
WCA & \\
Mobility model & Random waypoint \\
No. of nodes & 100 \\
Transmission range & $250(\mathrm{~m})$ \\
Size of network & $500^{*} 500(\mathrm{~m})$ \\
Pause time & $25(\mathrm{sec})$ \\
Hello interval & $5.0(\mathrm{sec})$ \\
Simulation duration & 500 times \\
\hline
\end{tabular}

\section{RESULTS AND DISCUSSION}

DCCHSM used a routed topology dataset for its implementation on NS2 simulating the proposed topology. The number of nodes taken in the requirement phase was 100 nodes to be grouped into five clusters. Figure 6 depicts the screen output of DCCHSM requirements Table 1. Each node communicates with other nodes directly or indirectly. The required number of nodes as specified in the input to DCCHSM is created. Figure 7 depicts DCCHSM's node creation.

Transportation networks with many nodes rise in complexity and their comparisons become difficult. A node's accessibility is not evident easily and measures and indices are needed to evaluate network efficiency. After nodes are created the average distances between the nodes are measured. DCCHSM's active distance measures helps in its efficient topology proposals based on time. A network with higher active distance tends to be less linked and active distances are reduced for higher connectivity (Planar networks have larger active distances due to intermediate stops between two distant nodes). Figure 8 depicts DCCHSM's active distance measure.

DCCHSM's connects nodes based on the minimum average distances between nodes for constructing the cluster groups and eventually the connectivity pattern. Figure 9 depicts DCCHSM's node connectivity pattern.

To validate its generated clustered pattern it uses the threshold value of beacons. The use of beacon inhibition is used to reduce collisions and increase transmission efficiencies as any node can emit many beacon messages based on the threshold value. DCCHSM estimates the threshold value based on Eq. 1 listed above and as the ratio between the range of the beacon and time taken to send the beacon. Figure 10 depicts the threshold value calculated in the network.

Nodes stop sensing when the total number of beacon messages is less than the threshold value, thus, reducing power consumption at the nodes. A node may waste energy when idle in listening or colliding or overhearing or in control. The proposed topology nodes communicate only to listening nodes or are in a sleep mode. Hibernation mechanisms determine nodes entering into a listening and the beacon interval. Table 2 lists the comparative performances in using $\mathrm{DCH}, \mathrm{WCA}$ and DCCHSM in terms of flooding times.

\begin{tabular}{lccc}
\multicolumn{4}{l}{ Table 2: Comparative flooding times in networks } \\
\hline No.of nodes & DCH & WCA & DCCHSMtime (sec) \\
\hline 20 & 0.513 & 0.354 & 0.211 \\
40 & 0.414 & 0.421 & 0.257 \\
60 & 0.654 & 0.452 & 0.354 \\
80 & 0.638 & 0.532 & 0.352 \\
100 & 0.761 & 0.698 & 0.301 \\
\hline
\end{tabular}




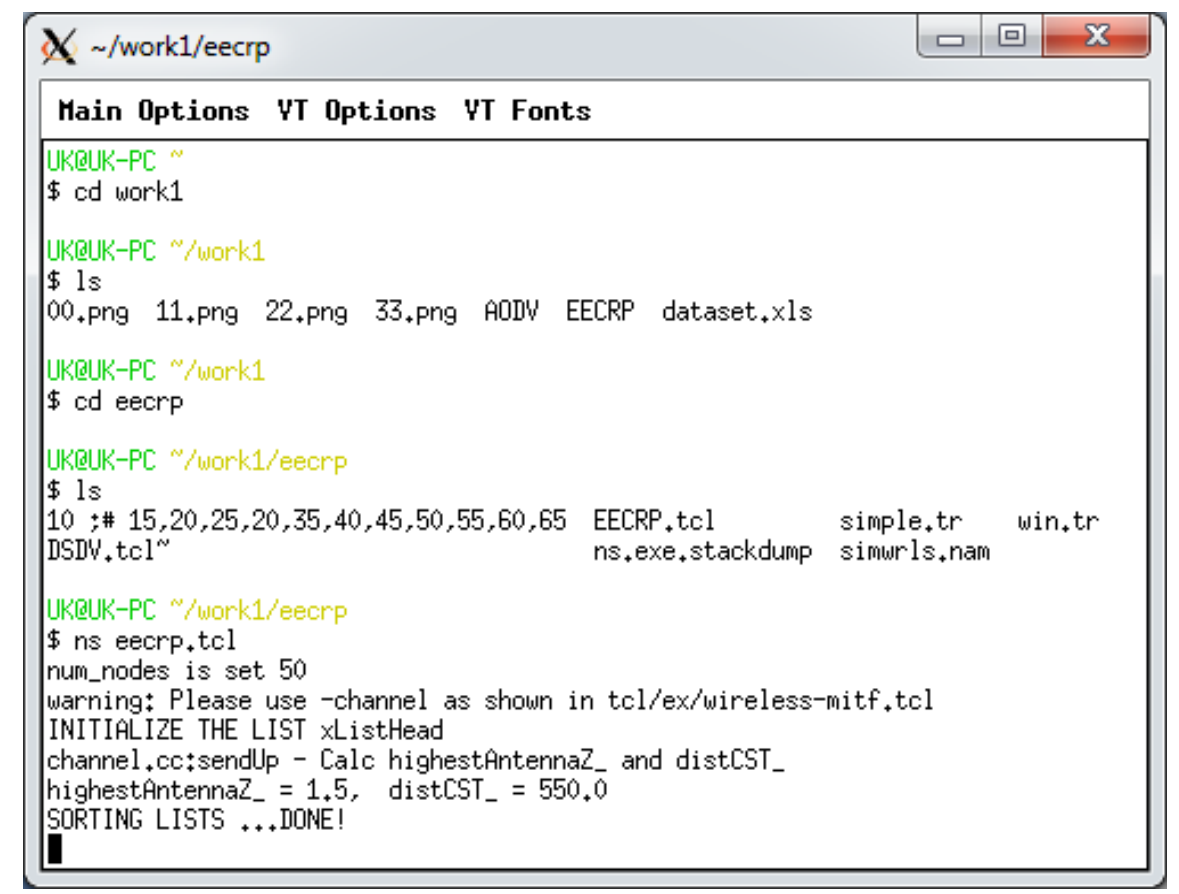

Fig. 6: DCCHSM requirements

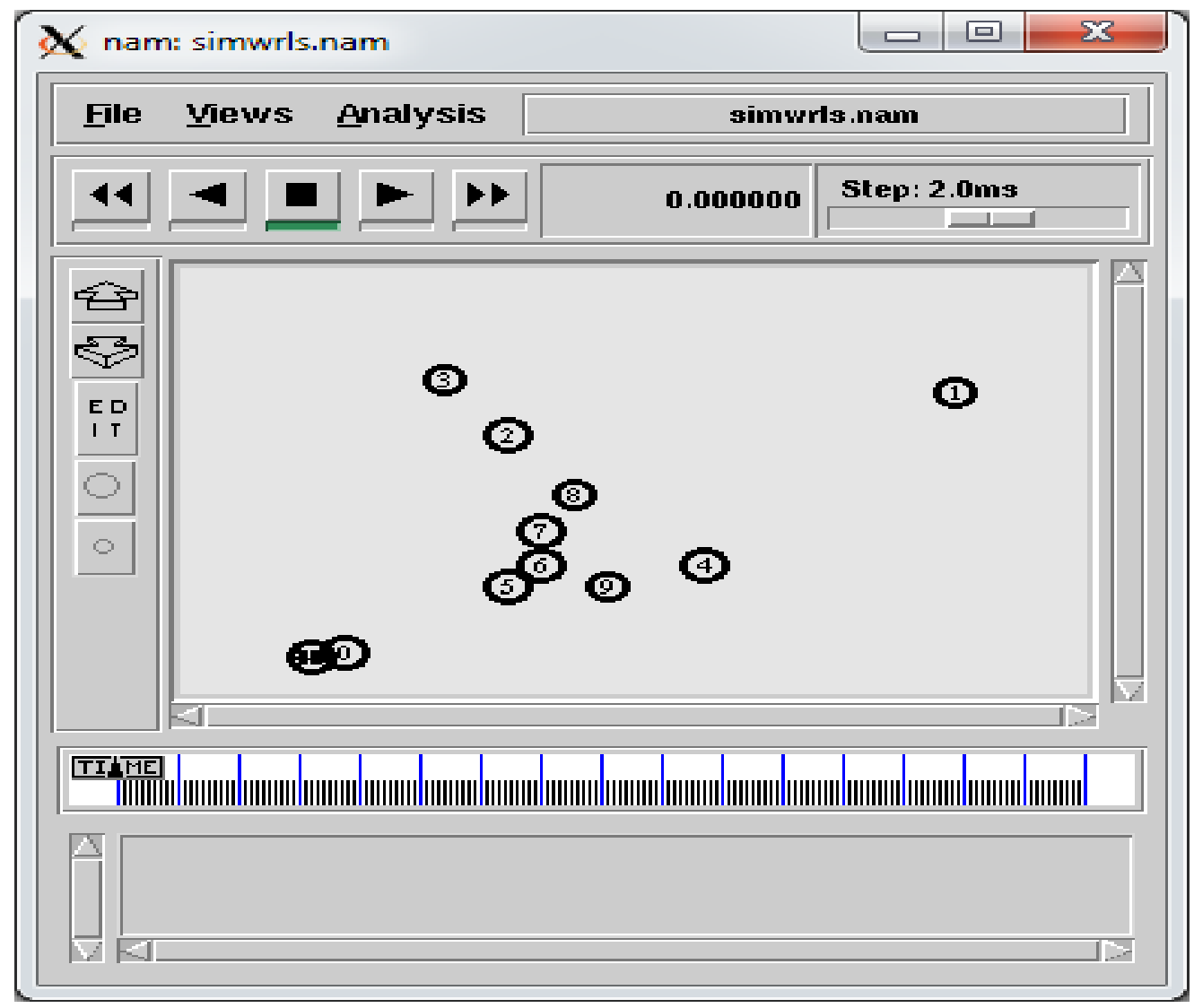

Fig. 7: DCCHSM node creation 
J. Eng. Applied Sci., 14 (15): 5287-5296, 2019

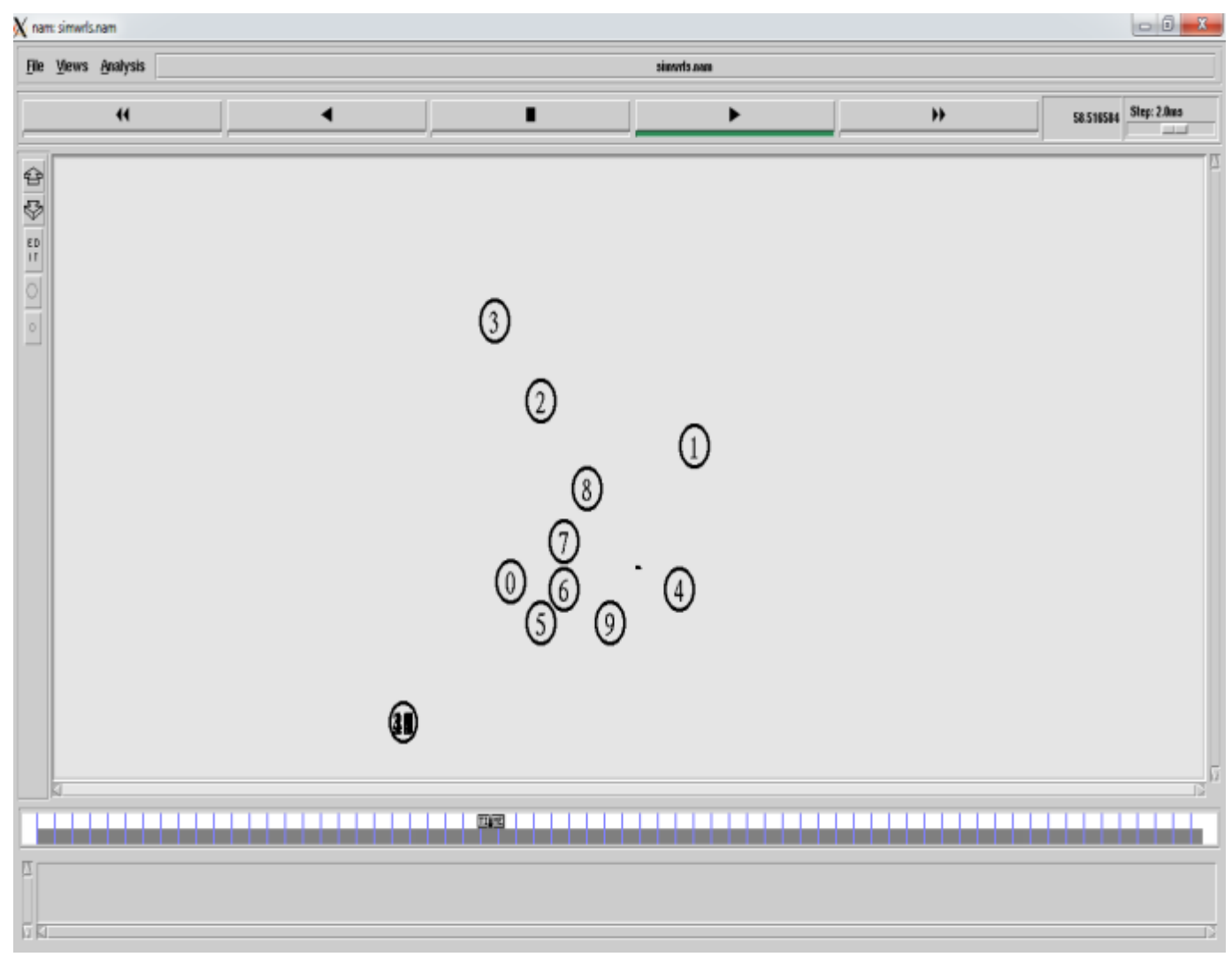

Fig. 8: DCCHSM active distance measure

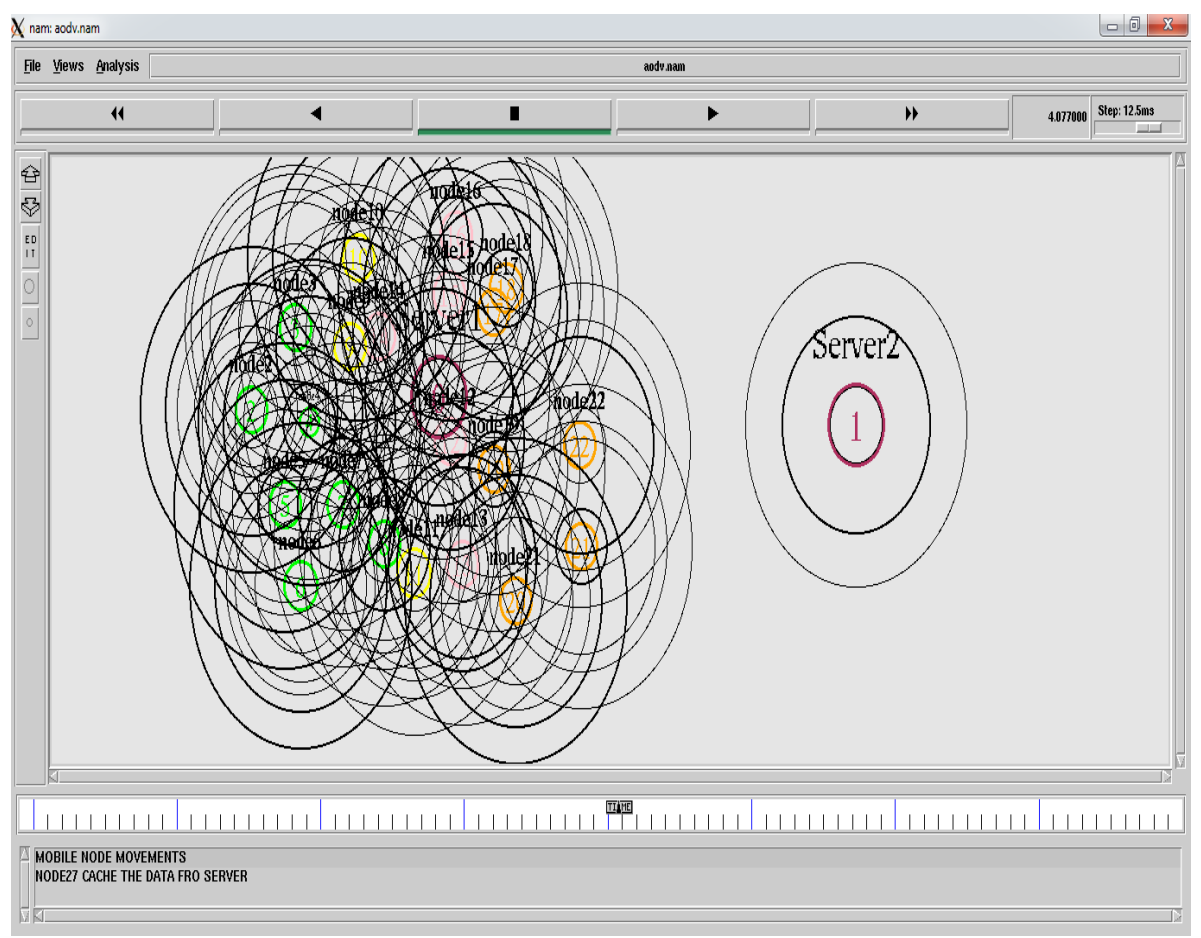

Fig. 9: DCCHSM node connectivity pattern 


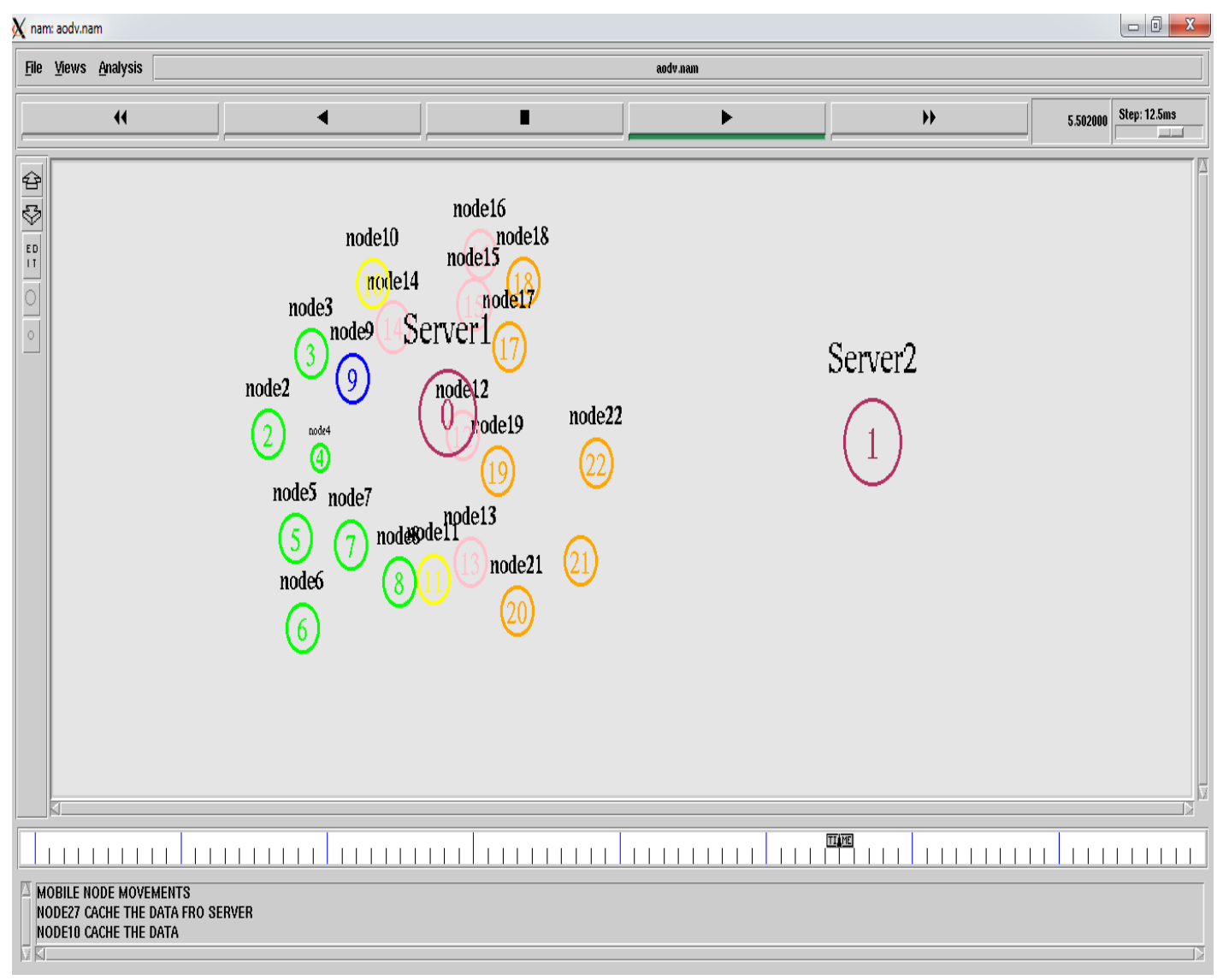

Fig. 10: DCCHSM threshold value

It is evident from the above table that DCCHSM's topology performs better as the network size grows complex. In comparison to the DCH flooding time of $0.761 \mathrm{sec}$ in 100 nodes, WCA based beacons consume lesser time in $0.698 \mathrm{sec}$ but DCCHSM consumes the least time with $0.278 \mathrm{sec}$ and is at least three times faster than WCA based techniques. Further on energy consumption it scores high when match up to directed diffusion techniques as depicted in Fig. 11.

DCCHSM advantages: Sensor nodes have low-end CPUs with limited memory. DCCHSM's customary routing consumes smallest processing overhead to make execution sufficient and effectual on low-end processor sensor nodes. A MANET is predictable to remain operational for an enlarged period of time (Raghavendra et al., 2004). The network and routing protocols in particular, must be empowered with self-organizing and self-management properties in order to let the network functioning as autonomic systems. DCCHSM has the capability to re-organize on its own based on the number of nodes deployed in a MANET.

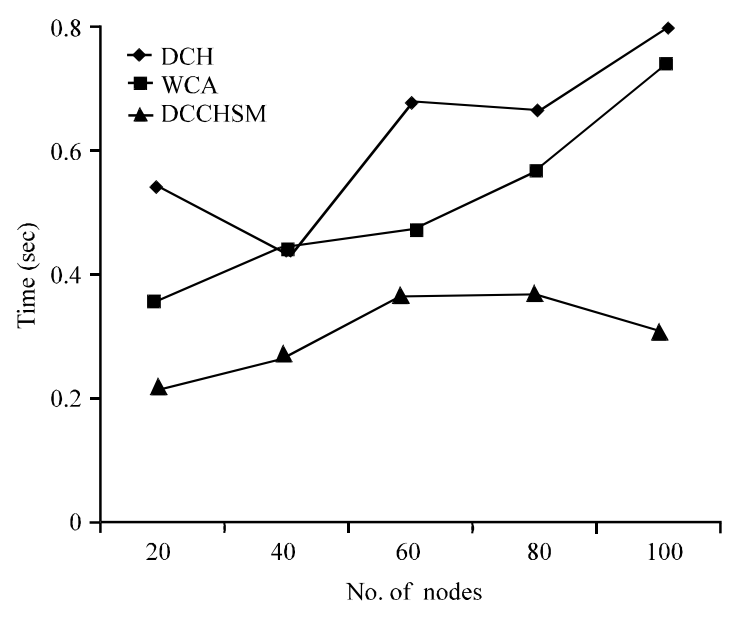

Fig. 11: DCCHSM energy consumption comparisons

Thisrouting protocol minimizes the No. of transmissions involved in path discovery and information delivery. It identifies shortest routes and cluster assignments for distributing and the forwarding of data packets across multiple paths, helping in an even depletion of 
node energy thus risingthe whole network duration. Thus, DCCHSM is an energy aware routing protocol. In a broad range of MANET applications, thousands or even millions of nodes participate and a routing protocol has tkjo cope with several challenges like countering radio interferences, encountering long pathsand handling unpredictable failures. It should also be able to display a scalable performance in spite of these hurdles. DCCHSM is scalable, since, it initially assesses a network and updates itself on re-configurations. A MANET can produce large quantity of locally redundant data as information is accessible to all nodes in the range. However, not allsolitary piece of information want to be communicated and a set of neighboring nodes can be incompletely aggregated and processed as close as probable to its origin. In this technique, it is possible to significantly condense the No. of transmissions, saving on the limited presented hardware resources and reducing the negative effects due to radio intrusion. DCCHSM effectively supports the setup and use of data routes for data aggregation. DCCHSM is also very reactive as paths are explored and associate only when required. In proactive DCCHSM, routing information for all recognized destinations is maintained up-to-date all the time, irrespective of whether a destination is being selected or not for data transmission. DCCHSM uses a permutation of two techniques and hence can be called a hybrid protocol (Royer and Toh, 1999; Di Caro et al., 2005 and Vijayalakshmi and Prabu, 2018a, b).

\section{CONCLUSION}

A wireless network is formed by connecting the nodes to a sink node which then further transmits the data to Base Station (BS) where the base station itself can work as a sink node. There are varieties of nodes within the network which are application dependent. The nodes collect information and forward the same to sink node which other forwards the message to a base station or server. Restrictions of node resources place a edge on the types of mechanisms used for routing and deployment. Therefore, a several routing protocols for MANETs have been developed in the last years. This study has presented a routing algorithm DCCHSM for MANETs. It has demonstrated a hybrid hierarchical energy aware routing protocol that can maximize speed, efficiency and be implemented in networks that are complex. It has attempted to overcome issues in topological segmentation methods. It has effectively used clustering to identify nearest nodes and grouped them for better productivity. The proposed scheme is scalable as segmentation results are optimized by the use thresholding on node locations. The performances of the proposed technique show that it can work even on complex networks. Since, the proposed method is based on mutual information share between neighbors, it increases the segmentation robustness and drives the architecture towards a more rational topological/semantic map. Though simulated on NS2, it can be adapted to real time network problems for increasing energy efficiency and speeds. It can be concluded that DCCHSM is a viable routable protocol that can be applied to existing networks for upgrades or while creating a new networks and is its architecture is scalable, robust and energy efficient.

\section{REFERENCES}

Abbasi, A.A. and M. Younis, 2007. A survey on clustering algorithms for wireless sensor networks. Comput. Commun., 30: 2826-2841.

Al-Karaki, J.N. and A.E. Kamal, 2004. Routing techniques in wireless sensor networks: A survey. IEEE Wireless Commun., 11: 6-28.

Basagni, S., I. Chlamtac, V.R. Syrotiuk and B.A. Woodward, 1998. A Distance Routing Effect Algorithm for Mobility (DREAM). Proceedings of the 4th Annual ACM/IEEE International Conference on Mobile Computing and Networking, October 25-30, 1998, Dallas, Texas, USA., pp: 76-84.

Bellman, R.E., 1957. Dynamic Programming. Princeton University Press, Princeton, New Jersey, USA.,.

Chen, T.W. and M. Gerla, 1998. Global state routing: A new routing scheme for ad-hoc wireless networks. Proceedings of the International Conference on Communications, Volume 1, Jun 7-11, 1998, Atlanta, GA., pp: 171-175.

Chiang, C.C., H.K. Wu, W. Liu and M. Gerla, 1997. Routing in clustered multihop mobile wireless networks with Fading channel. Proceedings of the IEEE Singapore International Conference on Networks, April 1997, Singapore, pp: 197-211.

Di Caro, G., F. Ducatelle and L.M. Gambardella, 2005. AntHocNet: An Adaptive nature-inspired algorithm for routing in mobile ad hoc networks. Eur. Trans. Telecommun., 16: 443-455.

Eichler, S. and C. Roman, 2006. Challenges of secure routing in MANETs: A simulative approach using AODV-SEC. Proceedings of the 2006 IEEE International Conference on Mobile $\mathrm{Ad} \mathrm{Hoc}$ and Sensor Systems, October 9-12, 2006, IEEE, Vancouver, Canada, ISBN:1-4244-0506-8, pp: 481-484. 
Jacquet, P., P. Muhlethaler, T. Clausen, A. Laouiti, A. Qayyum and L. Viennot, 2001. Optimized link state routing protocol for ad hoc networks. Proceedings of the 5th IEEE Multi Topic Conference, August 7, 2001, Springer, USA., pp: 62-68.

Johnson, D.B. and D.A. Maltz, 1996. Dynamic Source Routing in Ad Hoc Wireless Networks. In: Mobile Computing, Imielinski, T. and H.F. Korth (Eds.). Springer, Boston, Massachusetts, USA., ISBN: 978-0-7923-9697-0, pp: 153-181.

Ndlovu, A., 2015. Improved energy efficient AODV routing using k-means algorithm for cluster head selection. Intl. J. Comput. Sci. Mob. Comput., 4: 177-187.

Pearlman, M.R. and Z.J. Haas, 1999. Determining the optimal configuration for the zone routing protocol. IEEE J. Sel. Areas Commun., 17: 13951414.

Perkins, C.E. and E.M. Royer, 1999. Ad-hoc on-demand distance vector routing. Proceedings of the 2nd Workshop on Mobile Computing Systems and Applications, February 25-26, 1999, New Orleans, LA., pp: 90-100.
Perkins, C.E. and P. Bhagwat, 1994. Highly dynamic Destination-Sequenced Distance-Vector routing (DSDV) for mobile computers. Proceedings of the Conference on Communications Architectures, Protocols and Applications, August 31-September 2, 1994, England, UK., pp: 234-244.

Raghavendra, C.S., K.M. Krishna and T. Znati, 2004. Wireless Sensor Networks. 1st Edn., Springer, Berlin, Germany, ISBN:978-1-4020-7884-2, Pages: 426.

Royer, E.M. and C.K. Toh, 1999. A review of current routing protocols for ad hoc mobile wireless networks. IEEE Personal Commun., 6: 46-55.

Schiller, J.H., 2003. Mobile Communications. Addison-Wesley Company, Boston, Massachusetts, USA., ISBN:9780321123817, Pages: 492.

Vijayalakshmi, J. and K. Prabu, 2018a. A survey of various weighted based clustering algorithm for MANET. Intl. J. Data Technol. Appl., 7: 146-153.

Vijayalakshmi, J. and K. Prabu, 2018b. Performance analysis of clustering schemes in MANETs. Proceedings of the International Conference on Intelligent Data Communication Technologies and Internet of Things, August 7-8, 2018, Springer, Cham, Switzerland, ISBN:978-3-030-03145-9, pp: 808-813. 